\title{
LIMIT CYCLES OF A CLASS OF GENERALIZED LIÉNARD POLYNOMIAL EQUATIONS
}

\author{
JAUME LLIBRE ${ }^{1}$ AND AMMAR MAKHLOUF ${ }^{2}$
}

\begin{abstract}
We prove that the generalized Liénard polynomial differential system

$$
\dot{x}=y^{2 p-1}, \quad \dot{y}=-x^{2 q-1}-\varepsilon f(x) y^{2 n-1},
$$

where $p, q$ and $n$ are positive integers, $\varepsilon$ is a small parameter and $f(x)$ is a polynomial of degree $m$ can have $[m / 2]$ limit cycles, where $[x]$ is the integer part function of $x$.
\end{abstract}

\section{IntRoduCtion AND STATEMENT OF THE MAIN RESUlts}

In 1900 Hilbert [2] in the second part of his 16-th problem proposed to find an uniform upper bound for the number of limit cycles of all polynomial differential systems of a given degree, and also to study their distribution or configuration in the plane. The 16-th problem, except the one related with the Riemann hypothesis, seems to be the most elusive of Hilbert's problems. It has been one of the main problems in the qualitative theory of planar differential equations during the XX century. Until now it is not proved the existence of such an uniform upper bound. This problem remains open even for the polynomial differential systems of degree 2. However it is not difficult to see that any finite configuration of limit cycles is realizable for some polynomial differential system, see for details [6].

Following to Smale [8] many authors consider an easier and special class of polynomial differential systems, the Liénard polynomial differential equation:

$$
\ddot{x}+f(x) \dot{x}+x=0,
$$

where

$$
f(x)=a_{0}+a_{1} x+\ldots+a_{m} x^{m},
$$

2010 Mathematics Subject Classification. Primary 34C05, 34C25.

Key words and phrases. limit cycles, polynomial differential systems, Liénard systems, averaging theory, $(p, q)$-trigonometric functions. 
and the dot denotes derivative with respect to the time $t$. The Liénard polynomial equation (2) of second order can be written as the Liénard differential system of first order

$$
\dot{x}=y, \quad \dot{y}=-x-f(x) y .
$$

For these systems the existence of uniform bounds also remain unproved.

Here we want to study the number of limit cycles of the following generalized Liénard polynomial differential system of first order

$$
\dot{x}=y^{2 p-1}, \quad \dot{y}=-x^{2 q-1}-\varepsilon f(x) y^{2 n-1},
$$

where $p, q$ and $n$ are positive integers, $\varepsilon$ is a small parameter and $f(x)$ is the polynomial (3).

In fact system (4) with $\varepsilon=0$ is a Hamiltonian system with Hamiltonian

$$
H(x, y)=\frac{1}{2 q} x^{2 q}+\frac{1}{2 p} y^{2 p}
$$

This system has a global center at the origin of coordinates, i.e. the periodic orbits surrounding the origin filled the whole plane $\mathbb{R}^{2}$, and we want to study how many periodic orbits persist after perturbing the periodic orbits of this center as in the system (4) for $\varepsilon \neq 0$ sufficiently small.

Let $[x]$ denotes the integer part function of $x \in \mathbb{R}$. Our main result is the following one.

Theorem 1. For $\varepsilon \neq 0$ sufficiently small the polynomial differential system (4) can have at least $[\mathrm{m} / 2]$ limit cycles if $m$ is the degree of the polynomial $f(x)$.

Theorem 1 is proved in section 2 using averaging theory of first order. See the appendix for a summary of the results on averaging theory here used. Note that the maximum number of limit cycles obtained using the averaging theory of first order only depends on $m$ the degree of the polynomial $f(x)$, and it is independent of $p, q$ and $n$.

System (4) with $p=q=n=1$ was studied by Lins et al. [5] in 1977, and for $p=n=1$ and $q$ arbitrary has been studied by Urbino et al. [9] in 1993. Other generalizations of Liénard systems have also been studied, see for instance $[3,7]$.

\section{Proof of Theorem 1}

The key point in the proof of many results on differential systems is the election of good coordinates for studying the systems. 
Following Liapunov [4] we introduce the $(p, q)$-trigonometric functions $z(\theta)=\operatorname{Cs} \theta$ and $w(\theta)=\operatorname{Sn} \theta$ as the solution of the following initial value problem

$$
\dot{z}=-w^{2 p-1}, \quad \dot{w}=z^{2 q-1}, \quad z(0)=p^{-\frac{1}{2 q}}, \quad w(0)=0 .
$$

It easy to check that the functions $\operatorname{Cs} \theta$ and $\operatorname{Sn} \theta$ satisfy the equality

$$
p \operatorname{Cs}^{2 q} \theta+q \operatorname{Sn}^{2 p} \theta=1 .
$$

For $p=q=1$ we have that $\operatorname{Cs} \theta=\cos \theta$ and $\operatorname{Sn} \theta=\sin \theta$; i.e. the $(1,1)$-trigonometric functions are the classical ones. It is known that $\operatorname{Cs} \theta$ and $\operatorname{Sn} \theta$ are $T$-periodic functions with

$$
T=2 p^{-\frac{1}{2 q}} q^{-\frac{1}{2 p}} \frac{\Gamma\left(\frac{1}{2 p}\right) \Gamma\left(\frac{1}{2 q}\right)}{\Gamma\left(\frac{1}{2 p}+\frac{1}{2 q}\right)},
$$

where $\Gamma(x)$ is the Gamma function.

The $(p, q)$-polar coordinates $(r, \theta)$ are defined as

$$
x=r^{p} \operatorname{Cs} \theta, \quad y=r^{q} \operatorname{Sn} \theta .
$$

The generalized Liénard differential system $(4)$ in the $(p, q)$-polar coordinates becomes

$$
\begin{aligned}
& \dot{r}=-\varepsilon r^{q(2 n-2)+1} \operatorname{Sn}^{2(p+n-1)} \theta f\left(r^{p} \operatorname{Cs} \theta\right), \\
& \dot{\theta}=-r^{2 p q-p-q}-\varepsilon p r^{2 q(n-1)} \operatorname{Cs} \theta \operatorname{Sn}^{2 n-1} \theta f\left(r^{p} \operatorname{Cs} \theta\right) .
\end{aligned}
$$

Taking as independent variable the angular variable $\theta$ the differential system (6) writes

$$
\begin{aligned}
\frac{d r}{d \theta} & =-\varepsilon r^{(2 n-1) q+p+1-2 p q} \operatorname{Sn}^{2(p+n-1)} \theta f\left(r^{p} \operatorname{Cs} \theta\right)+O\left(\varepsilon^{2}\right), \\
& =\varepsilon F_{1}(\theta, r)+O\left(\varepsilon^{2}\right) .
\end{aligned}
$$

Now we shall apply the Theorem 2 of the appendix. Using the notation defined there we have

$$
\mathbf{x}=\mathbf{y}=r, \quad t=\theta, \quad F_{1}(t, \mathbf{x})=F_{1}(\theta, r) .
$$

Then, using (3) we have that

$$
\begin{aligned}
g(r) & =\sum_{k=0}^{m} a_{k} r^{(2 n-1) q+p+1-2 p q+p k} \int_{0}^{T} \operatorname{Sn}^{2(p+n-1)} \theta \mathrm{Cs}^{k} \theta d \theta \\
& =\sum_{k=0}^{m} a_{k} r^{(2 n-1) q+p+1-2 p q+p k} b_{k} .
\end{aligned}
$$


From [4] it follows that $b_{k}=0$ if $k$ is odd, and clearly $b_{k}>0$ if $k$ is even. So we have

$$
g(r)=r^{(2 n-1) q+p+1-2 p q} \sum_{\substack{k=0 \\ k \text { even }}}^{m} a_{k} b_{k} r^{p k} .
$$

We recall the Descartes Theorem about the number of zeros of a real polynomial (for a proof see for instance [1]).

Descartes Theorem. Consider the real polynomial $p(x)=a_{i_{1}} x^{i_{1}}+$ $a_{i_{2}} x^{i_{2}}+\cdots+a_{i_{k}} x^{i_{k}}$ with $0 \leq i_{1}<i_{2}<\cdots<i_{k}$ and $a_{i_{j}} \neq 0$ real constants for $j \in\{1,2, \cdots, k\}$. When $a_{i_{j}} a_{i_{j+1}}<0$, we say that $a_{i_{j}}$ and $a_{i_{j+1}}$ have a variation of sign. If the number of variations of signs is $m$, then $p(x)$ has at most $m$ positive real roots. Moreover, it is always possible to choose the coefficients of $p(x)$ in such a way that $p(x)$ has exactly $k-1$ positive real roots.

By Descartes Theorem the polynomial $g(r)$ has at most $[m / 2]$ positive roots $r$. Then, by Theorem 2 it follows that the differential equation $(7)$ can have $[m / 2]$ periodic solutions, and consequently the differential systems (6) or equivalently (4) can have $[\mathrm{m} / 2]$ periodic solutions which can be chosen alternatively stable and unstable. In short, the differential system $(4)$ can have $[m / 2]$ limit cycles. This completes the proof of Theorem 1.

\section{APPENDIX: AVERAGING THEORY OF FIRST ORDER}

We consider the initial value problems

$$
\dot{\mathbf{x}}=\varepsilon F_{1}(t, \mathbf{x})+\varepsilon^{2} F_{2}(t, \mathbf{x}, \varepsilon), \quad \mathbf{x}(0)=\mathbf{x}_{0},
$$

and

$$
\dot{\mathbf{y}}=\varepsilon g(\mathbf{y}), \quad \mathbf{y}(0)=\mathbf{x}_{0},
$$

with $\mathbf{x}, \mathbf{y}$ and $\mathbf{x}_{0}$ in some open $\Omega$ of $\mathbb{R}^{n}, t \in[0, \infty), \varepsilon \in\left(0, \varepsilon_{0}\right]$. We assume that $\mathbf{F}_{\mathbf{1}}$ and $\mathbf{F}_{\mathbf{2}}$ are periodic of period $\mathrm{T}$ in the variable $\mathrm{t}$, and we set

$$
g(\mathbf{y})=\frac{1}{T} \int_{0}^{T} F_{1}(t, \mathbf{y}) d t .
$$

Theorem 2. Assume that $F_{1}, D_{\mathbf{x}} F_{1}, D_{\mathbf{x x}} F_{1}$ and $D_{\mathbf{x}} F_{2}$ are continuous and bounded by a constant independent of $\varepsilon$ in $[0, \infty) \times \Omega \times\left(0, \varepsilon_{0}\right]$, and that $y(t) \in \Omega$ for $t \in[0,1 / \varepsilon]$. Then the following statements holds:

(1) For $t \in[0,1 / \varepsilon]$ we have $\mathbf{x}(t)-\mathbf{y}(t)=O(\varepsilon)$ as $\varepsilon \rightarrow 0$. 
(2) If $p \neq 0$ is a singular point of system (9) and $\operatorname{det} D_{\mathbf{y}} g(p) \neq$ 0 , then there exists a periodic solution $\phi(t, \varepsilon)$ of period $T$ for system (8) which is close to $p$ and such that $\phi(0, \varepsilon)-p=O(\varepsilon)$ as $\varepsilon \rightarrow 0$.

(3) The stability of the periodic solution $\phi(t, \varepsilon)$ is given by the stability of the singular point.

We have used the notation $D_{\mathbf{x}} g$ for all the first derivatives of $g$, and $D_{\mathrm{xx}} g$ for all the second derivatives of $g$.

For a proof of Theorem 2 see [10].

\section{ACKNOWLEDGMENTS}

The first author is partially supported by a MCYT/FEDER grant MTM2008-03437, a CIRIT grant number 2009SGR-410, an ICREA Academia, and FP7-PEOPLE-2012-IRSES 316338 and 318999.

\section{REFERENCES}

[1] I.S. Berezin AND N.P. Zhidkov, Computing Methods, Volume II, Pergamon Press, Oxford, 1964.

[2] D. Hilbert, Mathematische Probleme, Lecture, Second Internat. Congr. Math. (Paris, 1900), Nachr. Ges. Wiss. G"ttingen Math. Phys. KL. (1900), 253-297; English transl., Bull. Amer. Math. Soc. 8 (1902), 437-479.

[3] G. Kolutsky, An upper estimate for the number of limit cycles of even-degree Liénard equations in the focus case, J. Dyn. Control Syst. 17 (2011), 231-241.

[4] A.M. Liapunov, Stability of motion, in Mathematics in Science and Engineering, vol. 30, Academic Press, 1966.

[5] A. Lins, W. DE Melo And C.C. Pugh, On Liénard's Equation, Lecture Notes in Math. 597, Springer, Berlin, 1977, pp 335-357.

[6] J. Llibre AND G. Rodríguez, Configurations of limit cycles and planar polynomial vector fields, J. of Differential Equations 198 (2004), 374-380.

[7] M. Han, The Hopf cyclicity of Lienard systems, Appl. Math. Lett. 14 (2001), $183-188$

[8] S. Smale, Mathematical Problems for the Next Century, Mathematical Intelligencer 20, (1998), 7-15.

[9] A.M. Urbina, G.L. de la Barra, G. León, M.L. de la Barra And M. CAÑAS, Limit cycles of Liénard equations with nonlinear damping, Canad. Math. Bull. 36 (1993), 251-256.

[10] F. Verhulst, Nonlinear Differential Equations and Dynamical Systems, Universitext, Springer, New York, 1996.

${ }^{1}$ Departament de Matematiques, Universitat Autònoma de Barcelona, 08193 Bellaterra, Barcelona, Catalonia, Spain

E-mail address: jllibre@mat.uab.cat 
2 Department of Mathematics, University of Annaba, Elhadjar 23 Annaba, Algeria

E-mail address: makhloufamar@yahoo.fr 